\title{
Intensidad del entrenamiento personal dirigido en modalidades de danza y baile
}

\section{Intensity of directed personal training in dance modalities}

\author{
Elena Conesa Ros
}

Facultad de Ciencias del Deporte, Universidad de Murcia, Campus de Excelencia Internacional Regional "Campus Mare Nostrum".

\begin{abstract}
Resumen: Actualmente, la población adulta general utiliza multitud de modalidades deportivas, actividades dirigidas y medios de locomoción para realizar la actividad física diaria recomendada por los organismos e instituciones de referencia. Entre estas nuevas disciplinas ha surgido con fuerza en los últimos años diferentes modificaciones de actividades de baile de salón y bailes modernos. Para conocer si estas disciplinas exigen del participante la intensidad de esfuerzo recomendada por la literatura científica internacional, este trabajo analizó la intensidad relativa expresada como porcentaje de la frecuencia cardiaca máxima (\%FCmax) y de reserva (\%FCR) en los estilos de baile de Hip-Hop y Salsa con sesiones programadas y dirigidas por expertos.Siete jóvenes con experiencia en la práctica de actividades dirigidas con soporte musical realizaron 5 sesiones estandarizadas e iguales en volumen, intensidad y pasos para cada una de las dos modalidades sometidas a estudio. Los resultados muestran que el baile Hip-Hop tiene una exigencia de intensidad media y máxima que le permite catalogar como actividad vigorosa (\%FCR 64\% y $86 \%$ ), mientras que la Salsa debe catalogarse como intensidad moderada (\%FCR 57\% y 77\%). Aunque ambas modalidades cumplen los requisitos de intensidad recomendadapara la población adulta sana, la mayor intensidad relativa que presenta el Hip-Hop permite reducir el volumen semanal total a unos 75 min para alcanzar los objetivos cardiosaludables marcados, mientras que la Salsa requiere de al menos 150 minutos semanales.

Palabras Clave: Actividad Física, Deporte, expresión corporal, danza, fitness.
\end{abstract}

Abstract: Currently, the general adult population uses a multitude of sporting modalities, directed activities and locomotion modalities to carry out the daily physical activity recommended by the organisms and institutions of reference. Among these new disciplines has been emerged strongly in recent years different modifications of dance activities and modern dances. To determine if these disciplines require the intensity of effort recommended by the international scientific literature, this work analyzed the relative intensity expressed as a percentage of maximum heart rate (\%HRmax) and reserve (\%HRR) in dance styles of Hip-Hop and Salsa with programmed and expert-led sessions. Seven young people with experience in the practice of activities directed by musical support performed 5 standardized and equal sessions in volume, intensity and steps for each of the two modalities under study. The results show that Hip-Hop dance has a medium and maximum intensity requirement that allows it to be classified as vigorous activity (\%HRR 64\% and 86\%), whereas Salsa should be classified as moderate intensity (\%HRR 57\% and 77\% \%). Although both modalities meet the requirements of recommended intensity for the healthy adult population, the greater relative intensity of the Hip-Hop allows the total weekly volume to be reduced to about 75 minutes to reach the marked heart-healthygoals, whereas the Salsa requires at least 150 minutes per week.

Key-words: Physical Activity, Sport, body language, dance, fitness.

\section{Introducción}

El Colegio Americano de Medicina del Deporte (ACSM, 2011), quizás la institución de referencia y mayor prestigio internacional en el estudio y difusión de los efectos del ejercicio físico sobre la salud de la población general, ha concluido que las personas adultas sanas van a poder mejorar notablemente su calidad de vida realizando ejercicio físico supervisado durante 30-60 min al día, entre 3 y 5 días semanales, hasta alcanzar un total de 75-150 min de ejercicio de resistencia cardiorrespiratoria a la semana. Este amplio rango de volúmenes de entrenamiento propuesto, por día y por semana de entrenamiento, se debe a que la mayor o menor intensidad relativa a la que el individuo realice la actividad va a requerir de un menor o mayor volumen de entrenamiento respectivamente para alcanzar las adaptaciones cardiorrespiratorias deseadas. Así, dentro de la clasificación que la ACSM (2011)

Dirección para correspondencia [Correspodence address]: Elena Conesa Ros. Facultad de Ciencias del Deporte, Universidad de Murcia, C/ Argentina s/n 30720, Murcia (España).E-mail: econesaros@um.es hace de la intensidad de esfuerzo (Tabla1), ese posicionamiento recomienda que la intensidad de estos entrenamientos para personas adultas y aparentemente sanas se mantenga entre moderada y vigorosa. Aunque los rangos de intensidad vigorosa propuestas por esta institución y numerosas publicaciones de referencia (DiPietro, Dziura, Yeckel y Neufer, 2006; Swain, 2005) pudiesen parecer ya elevadas y exclusivamente reservadas para deportistas experimentados (Pallarés, MoránNavarro, Ortega, Fernández-Elías y Mora-Rodriguez, 2016), una nueva corriente de investigación parece postularse a favor del entrenamiento catalogado como máximo o casi máximo (Guadalupe-Grau et al., 2017; Helgerud, et al., 2007), o incluso supramaximo(intensidad de esfuerzo al 120-140\% del $\mathrm{VO}_{2 \max }$ ) (Burgomaster, Heigenhauser y Gibala, 2006; Gibala, Little, Macdonald yHawley, 2012) para la optimización de las adaptaciones cardiorrespiratorias de estos participantes con unos volúmenes de entrenamiento notablemente bajos ( 3 sesiones semanales de una duración de 20-25 min). 
Tabla 1. Clasificación de la intensidad del esfuerzo cardiorrespiratorio (Tomado de ACSM, 2011).

\begin{tabular}{lccc}
\hline \multicolumn{4}{c}{ Intensidad Relativa } \\
Intensidad & $\%$ FCR & $\%$ FCmax & $\begin{array}{c}\text { PSE } \\
\text { (escala 6-20) }\end{array}$ \\
Muy Liviana & $<30 \%$ & $<57 \%$ & $<9$ \\
Liviana & $30-39 \%$ & $57-63 \%$ & $9-11$ \\
Moderada & $40-59 \%$ & $64-76 \%$ & $12-13$ \\
Vigorosa & $60-89 \%$ & $77-95 \%$ & $14-17$ \\
Máximo o casi máximo & $>90 \%$ & $>96 \%$ & $>18$ \\
\hline \%FCR: \% de frecuencia cardiaca de reserve; \% FCmax: \% de la frecuencia cardiaca \\
máxima; PSE: percepción subjetiva del esfuerzo. \\
\hline
\end{tabular}

Desde principios del siglo XX, la actividad física con soporte musical y dirigida por entrenadores expertos como el aerobic, acuagym, ciclo-indoor o la tonificación dirigida ha emergido como una alternativapara producir adaptaciones cardiorrespiratorias positivas de forma lúdica y motivante (Bardal, Roeleveld, y Mork, 2015; Saavedra, De La Cruz, Escalante, y Rodríguez, 2007; Stanforth, Stanforth y Hoemeke, 2000) en contraposición al entrenamiento tradicional basado en la carrera, nado o pedaleo en modo continuo o interválico (García-Pallarés, Sánchez-Medina, Carrasco, Díaz, y Izquierdo, 2009; Helgerud et al., 2007; Pallarés y Morán-Navarro, 2012). Con este mismo propósito, actividades de danza y baile tradicionales o modernos como la Salsa, Zumba o HipHop, que requieren un gran componente técnico y de ritmo, se han adaptado en los últimos años a las características de estas actividades dirigidas del fitness y wellness, donde más que un objetivo estético y competitivo se busca obtener las adaptaciones cardiorrespiratorias y de control del peso recomendadas para la población adulta. Aunque estas nuevas modalidades de danza y baile en sesiones supervisadas por técnicos expertos pueden tener similares características motivaciones que las actividades dirigidas con soporte musical a través del ritmo y la música, hasta la fecha, existe un relatico vacío de conocimiento empírico sobre la grado de cumplimento de estas modalidades con la carga de entrenamiento que nos recomiendan las instituciones de referencia, especialmente en lo relativo a la intensidad (Donath, Roth, Hohn, Zahner y Faude, 2014). Una vez estudiada la exigencia media y máxima de estas actividades, pueden establecerse recomendaciones generales sobre el volumen diario y semanal de estas prácticas que permitan alcanzar los mínimos de ejercicio físico recomendado.

Por ello, el objetivo de este estudio fue identificar la intensidad relativa que producen en personas adultas, sanas y moderadamente experimentadas sesiones estandarizadas de las modalidades de baile adaptados al fitness y wellness de Salsa y Hip-Hop. La hipótesis que se establece es que ambas actividades de baile van a alcanzar como máximo una inten- sidad media de sesión catalogada como moderada (i.e., FCR 40-59\%; FCmax64-76\%), por lo que será necesario cumplir 5 sesiones de entrenamiento semanal (50-60 min/día) para alcanzar los niveles de actividad física mínimos requeridos.

\section{Método}

\section{Muestra}

Siete jóvenes adultos sanos con edades comprendidas entre los 15 y 28 ańos, con una media de edad de 20,3 $\pm 4,3$ ańos (2 varones y 5 mujeres) tomaron parte de forma voluntaria en el estudio. Todos los participantes presentaban una experiencia notable en la práctica de ejercicio físico regular $(15,2 \pm$ 3,3 años) y especialmente en la práctica de baile en cualquier modalidad (13,1 \pm 6,1 años). Ninguno de estos participantes tenía limitaciones físicas o lesiones musculo-esqueléticas que pudieran afectar el proceso de entrenamiento. Además, todos los participantes fueron informados en detalle acerca de los procedimientos experimentales y los posibles riesgos y beneficios de esta investigación. El estudio, que cumple con la Declaración de Helsinki, fue aprobado por el Comité de Bioética de la Universidad de Murcia, y se obtuvo el consentimiento informado por escrito de todos los atletas antes de comenzar la fase experimental.

\section{Diseño Experimental}

Tras una sesión de familiarización que se utilizó para explicar y practicar la estructura y pasos específicos de cada baile, así como la colocación y uso del monitor de frecuencia cardiaca (Polar RS400, Finlandia), todos los participantes se sometieron a 5 sesiones de entrenamiento en cada baile de forma aleatorizada y randomizada con 48-72 h de recuperación entre sesiones. El monitor de frecuencia cardiaca grabó un dato cada 5 segundos de pulso para su posterior análisis. Las sesiones estandarizadas de cada baile mantuvieron la siguiente estructura: i) 5 minutos de movilidad articular; ii) 5 minutos de carrera continua a baja intensidad; iii) $30 \mathrm{~min}$ de parte principal con 10 pasos específicos de cada baile (i.e., Hip-Hop o Salsa), con 2 minutos por cada paso y 1 minuto de recuperación entre pasos. La música escogida para cada tipo de baile presentaba una cadencia de 130 beats/min y 100 beats/min para el Hip-Hop y la Salsa respectivamente; iv) finalmente, todos los participantes realizaban $5 \mathrm{~min}$ de vuelta a la calma con estiramientos suaves y ejercicios de relajación.

Durante la semana previa a la intervención, la frecuencia cardiaca de reposo de cada participante se estableció como el valor medio de 3 determinaciones (3 días consecutivos) de frecuencia cardiaca en decúbito supino, nada más despertarse, sin haber ingerido ningún tipo de alimento, y tras 10 minutos de haberse colocado el pulsómetro. La frecuencia cardiaca 
máxima teórica de cada participante se obtuvo de la ecuación de estimación de Whaley, Kaminsky, Dwyer, Getchell y Norton (1992).

Fórmula para hombres:

FCmax $\left(\right.$ lat $\left.\cdot \mathrm{min}^{-1}\right)=203,9-[0,812 *$ edad $]+[0,276 *(\mathrm{FC}$ reposo $)]-\left[0,084^{*}\right.$ peso corporal $\left.)\right]-\left[4,5^{*}\right.$ (código fumar) $]$

Fórmula para mujeres:

FCmax $\left(\right.$ lat $\left.\cdot \mathrm{min}^{-1}\right)=204,8-[0,718 *$ edad $]+[0,162 *(\mathrm{FC}$ reposo) $]-[0,105 *$ peso corporal $)]-[6,2 *$ (código fumar) $]$

${ }^{*}$ Nota: Fuma $=1$ No Fuma $=0$

Además, para el cálculo del porcentaje de frecuencia cardiaca de reserva (FCR) a la que habían realizado la actividad se empleó la fórmula de Karvonen, Kentala y Mustala, (1957).

$\%$ FCReserva $=($ FCetto - FCreposo $) /($ FCmax - FCreposo $)$

\section{Análisis Estadístico}

Se emplearon métodos estadísticos estándar para el cálculo de las medias y la desviación estándar. Una vez confirmada la distribución normal de la muestra mediante la prueba de Shapiro-Wilk, se examinaron las diferencias entre las modalidades de Salsa y Hip-Hop para los valores de FCR y FCmax mediante una prueba t de Student para muestras independientes. En todo caso se consideró un nivel de significación estadística del 5\% ( $\mathrm{p} \leq 0.05)$. Para este análisis estadístico se empleó el paquete informático SPSS para Windows (versión 17.0, SPSS Inc., Chicago, Ilinois, EEUU).

\section{Resultados}

La tabla 2 muestra los resultados en valor absoluto de la frecuencia cardiaca media (FCmedia), máxima (FCmax) y mínima (FCmin) promedio de las cinco sesiones realizadas para cada baile. Se observan valores significativamente superiores de FCmedia y FCmax alcanzadas durante la sesión de HipHop, en comparación con el baile de Salsa.

Tabla 2. Valores absolutos de frecuencia cardiaca media, máxima y mínima para ambos tipos de baile y calentamiento.

\begin{tabular}{llll}
\hline & Calentamiento & Hip-Hop & Salsa \\
FCmedia (lat/min) & $120 \pm 12$ & $147 \pm 11$ & $137 \pm 13^{*}$ \\
FCmax (lat/min) & $149 \pm 16$ & $179 \pm 8$ & $165 \pm 14^{*}$ \\
FCmin (lat/min) & $87 \pm 10$ & $102 \pm 14$ & $105 \pm 11$ \\
\hline${ }^{*}$ Diferencias significativas entre Salsa y Hi-Hop. & &
\end{tabular}

SPORT TK: Revista Euroamericana de Ciencias del Deporte
Por otro lado, la figura 1 muestra los valores de frecuencia cardiaca alcanzada por estos sujetos en ambos bailes una vez expresados como \%FCReserva. De nuevo, los valores de intensidad del esfuerzo relativo evidencias valores significativamente superiores en el baile de Hip-Hop (\%FCRmedia 67\% y \%FCRmax 87\%), en comparación con el baile de Salsa (\%FCRmedia 57\% y \%FCRmax 77\%.).

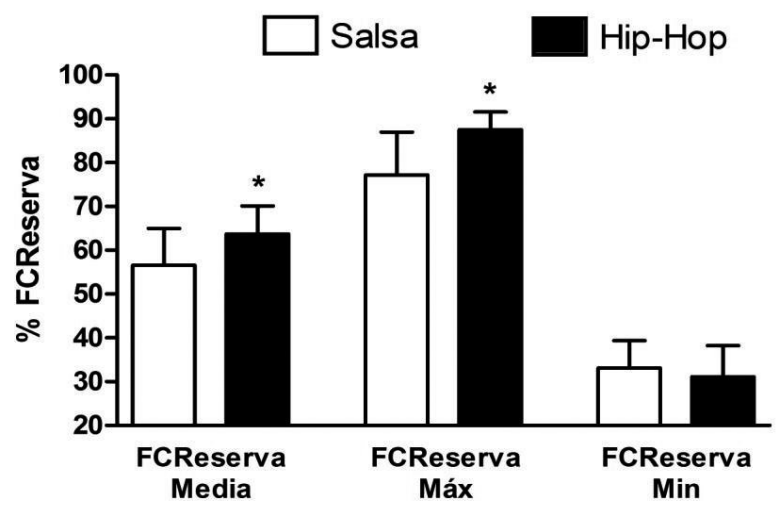

Figura 1. Valores de frecuencia cardiaca de reserva media, máxima y mínima de todas las sesiones de entrenamiento monitorizadas entre la disciplina de Salsa y Hip-Hop. Los datos se presentan como media y desviación estándar.

\section{Discusión}

Este estudio fue diseñado para conocer la intensidad relativa que representan en participantes adultos las nuevas adaptaciones o modificaciones de bailes clásicos y modernos en el contexto del fitness y wellness. Los principales hallazgos de este estudio nos indican que el valor de la magnitud de la intensidad media y máxima del baile Salsa permite catalogarla como actividad moderada ( $<60 \%$ FCR), y por contra, la modalidad de Hip-Hop representa una intensidad Vigorosa (>60\% FCR) (ACSM, 2011). Cabe destacar igualmente que la modalidad de Hip-Hop, además de tener una intensidad media de sesión vigorosa, lleva la participante en determinados momentos de la sesión a \%FCR próximos a valores máximos o casi máximos.

La clasificación del Hip-Hopcomo actividad vigorosa (i.e., FCR entre 60\% y 89\%) supone que cualquier individuo involucrado en esta modalidad va a requerir llevar a cabo únicamente tres sesiones semanales de similares características a las descritas en este estudio, es decir, con una duración aproximada de 45 min, incluidos calentamiento y vuelta a la calma, para cumplir con los mínimos requerimientos de ejercicio físico recomendado por el Colegio Americano de Medicina del Deporte (ACSM, 2011) para adultos sanos. Estamagnitud de esfuerzo físico permitirá a los participantes mejorar significa- 
tivamente su calidad de vida, reduciendo un buen número de factores de riesgo de accidente cardiovascular como la obesidad, la hipertensión o la diabetes tipo II (Guadalupe-Grau et al., 2017). Por contra, similares demandas energéticas y adaptaciones cardiorrespiratorias sólo se podrán alcanzar con la modalidad de Salsa si el participante realiza 4-5 sesiones semanales de intensidad equivalente, hasta alcanzar un volumen total semanal próximo a los $150 \mathrm{~min}$.

Para este estudio se han analizado las intensidades relativas a la frecuencia cardiaca máxima (\%FCmax) que representa cada tipo de esfuerzo en los participantes, pero también, una vez conocida la frecuencia cardiaca de reposo, se ha expresado la intensidad de forma relativa a la frecuencia cardiaca de reserva (\%FCR). La monitorización y programación individualizada del entrenamiento de resistencia cardiorrespiratoria mediante la \%FCR se ha mostrado superior en calidad metodológica respecto a la \%FCmax, puesto que diversos estudios han encontrado relaciones (i.e., correlaciones bivariadas) superiores entre el porcentaje del consumo de oxígeno de reserva $\left(\% \mathrm{VO}_{2} \mathrm{R}\right)$, conocido como el patrón de oro o Gold Standard del control de la intensidad mediante carga interna, y la \%FCR en comparación con la \%FCmax (Pallarés et al., 2016; Mora-Rodriguez, 2009; Morán-Navarro et al., 2016; Swain y Leutholtz, 1995; Swain, Leutholtz, King, Haas, y Branch, 1998). Estas investigaciones han evidenciado cómo la programación de la intensidad por medio del \%FCmaxinfraestima la intensidad real (\%VO2R) que está sosteniendo un deportista bien entrado y sobreestima la del desentrenado, puesto que no tiene en cuenta su frecuencia cardiaca de reposo y, por ende, su verdadero valor de gasto cardiaco de reserva. Igualmente, ya que no era recomendable someter a estos participantes a una prueba de rendimiento máxima para localizar la FCmax real, ha sido necesario estimar o predecir este valor. Para ello, en esta investigación se ha utilizado la ecuación validada por Whaleyet al. (1992), que ha mostrado mayor capacidad predictiva con respecto a otras ecuaciones de estimación como la de Karvonen de 1957 de 220-edad (Marins y Delgado Fernádez, 2007; Whaley, Kaminsky, Dwyer, Getchell, y Norton, 1992). Estas dos consideraciones metodológicas permiten tener mayor confianza en los resultados que hemos obtenido, especialmente en los relativo a la magnitud real de la intensidad del esfuerzo cardiorrespiratorio que representan ambas modalidades de baile en los participantes.

Investigaciones previas han evaluado la intensidad del esfuerzo en valor absoluto (i.e., pulsaciones por minuto) que producen otras actividades de baile. Así, por ejemplo, González, Vargas, Fernández, González, Gómez, y Costa (2011) encontraron que en el baile flamenco la FC media alcanzada fue de $159 \pm 13$ latidos por minuto para las mujeres, y de $155 \pm 12$ latidos por minuto para los hombres. Estos resultados nos aportan poca información útil, puesto que los valores de FC en términos absolutos no nos permiten conocer la intensidad relativa que cada individuo soportaba con estas actividades.

Ya en otras modalidades de fitness y wellness con soporte musical, pero alejadas del baile, estudios previos como el de López-Miñarro y Muyor (2010) y Muyor y López-Miñarro (2012) encontraron que los participantes de sesiones de cicloindoor mantenían FCR media del $74 \%$ y del $78 \%$ respectivamente. Posteriormente Muyor (2013) detectó de nuevo en la actividad del ciclo-indoor que el porcentaje de trabajo sobre la FCR estaría en el 80,6 $\pm 7,1 \%$, y que la percepción subjetiva del esfuerzo de estos individuos moderadamente activos, según la escala de BORG (6-20), alcanzaba los 14,9 $\pm 1,1$ puntos. Szabo, Gáspár, Kiss, y Radványi, (2015) realizaron un estudio con 18 mujeres en el que comparaban las diferencias de fatiga percibida mediante esta misma escala de Borg entre sesiones de ciclo-indoor con música y un técnico que dirige y motiva durante la actividad, o esta misma carga de trabajo, pero sin música ni técnico. Los resultados revelaron que los efectos y adaptaciones al entrenamiento eran similares en ambos casos, pero la percepción de fatiga y predisposición de las mujeres analizadas era mucho más positiva hacia un entrenamiento dirigido por un monitor y ambientado con una música de fondo. Estos resultados muestran que el usuario percibe una menor intensidad del esfuerzo de la que realmente realiza (\%FCR), hallazgo que bien puede ser extrapolado a los bailes analizados en esta investigación, ya que también son realizados con una base musical y un monitor.

\section{Conclusiones}

Teniendo en cuenta los resultados obtenidos puede concluirse que: a) se produce una mayor intensidad relativa en el baile de Hip-Hop que en la salsa, llegando esta última a considerarse de carácter vigorosa; b) ambas modalidades cumplen los requisitos de intensidad recomendada por la ACSM (2011) para la población adulta sana, pudiendo reducir el volumen semanal total del baile Hip-Hop a unos 75 min para alcanzar los objetivos cardiosaludables marcados, mientras que para la Salsa se requiere de al menos 150 minutos semanales.

\section{Referencias}

1. American College of Sports Medicine. (2011). ACSM Quantity and Quality of Exercise for Developing and Maintaining Cardiorespiratory, Musculoskeletal, and Neuromotor Fitness in Apparently Healthy Adults: Guidance for Prescribing Exercise. Medicine Science Sports Exercise, 43(7), 1334-1359.
2. Bardal, E. M, Roeleveld, K., \& Mork, P. J. (2015). Aerobic and cardiovascular autonomic adaptations to moderate intensity endurance exercise in patients with fibromyalgia. Journal Rehabilitation Medicine, 47(7), $639-46$.

3. Burgomaster, K. A., Heigenhauser, G. J., \& Gibala, M. J. (2006). Effect 
of short-term sprint interval training on human skeletal muscle carbohydrate metabolism during exercise and time-trial performance. Journal of Applied Physiology, 100(6), 2041-2047.

4. DiPietro, L., Dziura, J., Yeckel, C. W., \& Neufer, P. D. (2006). Exercise and improved insulin sensitivity in older women: evidence of the enduring benefits of higher intensity training. Journal of Applied Physiology, 100(1), 142-9.

5. Donath, L, Roth, R, Hohn, Y, Zahner, L, \& Faude O. (2004). The effects of Zumba training on cardiovascular and neuromuscular function in female college students. European Journal Sport Science, 14(6), 569-77.

6. Saavedra, J. M, De La Cruz, E, Escalante, Y, \&Rodríguez, F. A. (2007). Influence of a medium-impact aquaerobic program on health-related quality of life and fitness level in healthy adult females. Journal Sports Medicine Physiology Fitness, 47(4), 468-74.

7. García-Pallarés, J., Sánchez-Medina, L., Carrasco, L., Díaz, A., \&Izquierdo, M. (2009). Endurance and neuromuscular changes in worldclass level kayakers during a periodized training cycle. European Journal of Applied Physiology, 106(4), 629-638.

8. Gibala, M. J., Little, J. P., Macdonald, M. J., Hawley, J. A. (2012). Physiological adaptations to low-volume, high-intensity interval training in health and disease. The Journal of Physiology, 590(5), 1077-1084.

9. González, J. L., Vargas, A., Fernández, J. D. R., González, A., Gómez, R., \& Costa, J. L. (2011). Análisis del baile flamenco: cargas de trabajo y condición física. Revista Internacional de Medicina y Ciencias de la Actividad Fisica y del Deporte, 44, 708- 720.

10. Greco, C. C., Oliveira, A. S., Pereira, M. P., Figueira, T. R., Ruas, V. D., Goncalves, M., \&Denadai, B. S. (2011). Improvements in metabolic and neuromuscular fitness after 12 -week Bodypump ${ }^{\circ}$ training. The Journal of Strength \& Conditioning Research, 25(12), 3422-3431.

11. Guadalupe-Grau, A., Fernández-Elías, V. E., Ortega, J. F., Dela, F., Helge, J. W., \& Mora-Rodríguez, R. (2017). Effects of 6 months aerobic interval training on skeletal muscle metabolism in middle-aged metabolic syndrome patients. Scandinavian Journal Medicine Science Sports.doi: 10.1111/sms.12881. [Epub ahead of print]

12. Helgerud, J., Høydal, K., Wang, E., Karlsen, T., Berg, P., Bjerkaas, M., Simonsen, T., Helgesen, C., Hjorth, N., Bach, R., \&Hoff, J. (2007). Aerobic high-intensity intervals improve VO2max more than moderate training. Medicine Science Sports Exercise, 39(4), 665-71.

13. Karvonen, M. J., Kentala, E., Mustala, O. (1957). The effects of training on heart rate; a longitudinal study. Annales Medicinae Experimentalis et Biologiae Fenniae, 35(3), 307-315.

14. López-Min̄arro, P. A., \& Muyor, J. M. (2010). Heart rate and overall ratings of perceived exertion during Spinning ${ }^{\oplus}$ cycle indoor session in novice adults. Science \& Sports, 25(5), 238-244.

15. Mora-Rodríguez, R. (2009). Fisiología del deporte y el ejercicio: prácticas de campo y laboratorio. Ed. Médica Panamericana.

16. Marins, J., C., \& Delgado Fernández, M. (2007). Empleo de ecuacio- nes para predecir la frecuencia cardiaca máxima en carrera para jóvenes deportistas. Archivos de Medicina del Deporte, 24, 112-120.

17. Muyor, J. M. (2013). Exercise intensity and validity of the ratings of perceived exertion (Borg and OMNI Scales) in an indoor cycling session. Journal of Human Kinetics, 39(1), 93-101.

18. Muyor, J. M., \& Lopez-Miñarro, P. A. (2012). Overall ratings of perceived exertion and heart rate during indoor cycling session in non expert subjects. Medicina Dello Sport, 65(2), 145-154.

19. Oliveira, A. S., Greco, C. C., Pereira, M. P., Figueira, T. R., de Araújo Ruas, V. D., Gonçalves, M., \& Denadai, B. S. (2009). Physiological and neuromuscular profile during a bodypump session: Acute responses during a high-resistance training session. The Journal of Strength o Conditioning Research, 23(2), 579-586.

20. Pallarés, J. P., \&Morán-Navarro, M. Methodological approach to the cardiorespiratory endurance training. Journal of Sport and Health Research, 4(2), 119-136.

21. Pallarés, J. G., Morán-Navarro, R., Ortega, J. F., Fernández-Elías, V. E., \& Mora-Rodriguez, R. (2016). Validity and Reliability of Ventilatory and Blood Lactate Thresholds in Well-Trained Cyclists. PloS one, 11(9), e0163389.

22. Stanforth, D., Stanforth, P. R., \& Hoemeke, M. E. (2000). Physiologic and Metabolic Responses to a Body Pump Workout. The Journal of Strength \& Conditioning Research, 14(2), 144-150.

23. Szabo, A., Gáspár, Z., Kiss, N., \& Radványi, A. (2015). Effect of spinning workouts on affect. Journal of Mental Health, 24(3), 145-149.

24. Swain, D. P. (2005). Moderate or vigorous intensity exercise: which is better for improving aerobic fitness? Preventive Cardiology, 8(1), 55-58.

25. Swain, D. P., Leutholtz, B. C., King, M. E., Haas, L. A., \& Branch, J. D. (1998). Relationship between \% heart rate reserve and \% VO2 reserve in treadmill exercise. Medicine and Science in Sports and Exercise, 30(2), 318-321.

26. Swain, D. P., \& Leutholtz, B. C. (1997). Heart rate reserve is equivalent to \%VO2 reserve, not to \%VO2max. Medicine and Science in Sports and Exercise, 29(3), 410-414.

27. Sylta, Ø., Tønnessen, E., Hammarström, D., Danielsen, J., Skovereng, K., Ravn, T., \& Seiler, S. (2016). The effect of different high-intensity periodization models on endurance adaptations. Medicine \& Science in Sports \& Exercise, 48(11), 2165-2174.

28. Vargas, A. (2009). Danza y condición física. Revista del Centro de Investigación Flamenco Telethusa, 2(2), 16-24.

29. Whaley, M. H., Kaminsky, L. A., Dwyer, G. B., Getchell, L. H., \& Norton, J. A. (1992). Predictors of over-and underachievement of agepredicted maximal heart rate. Medicine and Science in Sports and Exercise, 24(10), 1173-1179.

30. Wyon, M. A., Abt, G., Redding, E., Head, A., \& Sharp, N. C. C. (2004). Oxygen uptake during modern dance class, rehearsal, and performance. The Journal of Strength \& Conditioning Research, 18(3), 646-649. 